Supplement of Biogeosciences Discuss., 12, 16285-16312, 2015

http://www.biogeosciences-discuss.net/12/16285/2015/

doi:10.5194/bgd-12-16285-2015-supplement

(C) Author(s) 2015. CC Attribution 3.0 License.

(c) (1)

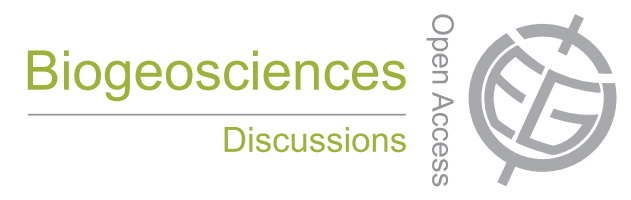

Supplement of

\title{
Carbon storage in seagrass soils: long-term nutrient history exceeds the effects of near-term nutrient enrichment
}

\author{
A. R. Armitage and J. W. Fourqurean \\ Correspondence to: A. R. Armitage (armitaga@tamug.edu)
}

The copyright of individual parts of the supplement might differ from the CC-BY 3.0 licence. 
Figure 2

\begin{tabular}{lcccccccc}
\multicolumn{2}{c}{ Aboveground seagrass carbon $(\mathrm{g} / \mathrm{m} 2)$} & & & & & \\
Site & Control & Control SE & Nitrogen & Nitrogen SE & Phosphorus & Phosphorus SE & N+P & N+P SE \\
Duck & 4.4 & 1.1 & 8.0 & 1.6 & 14.7 & 1.2 & 22.8 & 4.4 \\
S. Nest & 11.3 & 1.6 & 10.5 & 1.8 & 32.2 & 3.2 & 31.2 & 3.3 \\
Bob Allen & 4.8 & 1.6 & 2.6 & 0.5 & 29.3 & 6.1 & 34.8 & 5.3 \\
Rabbit & 42.3 & 3.7 & 46.5 & 5.3 & 33.8 & 5.1 & 49.4 & 9.2 \\
Nine Mile & 31.7 & 6.2 & 48.3 & 3.0 & 32.0 & 4.0 & 38.5 & 6.6 \\
Sprigger & 15.2 & 2.1 & 28.5 & 8.8 & 21.9 & 4.5 & 29.0 & 2.9
\end{tabular}

\begin{tabular}{|c|c|c|c|c|c|c|c|c|}
\hline \multicolumn{9}{|c|}{ Belowground seagrass carbon $(\mathrm{g} / \mathrm{m} 2)$} \\
\hline Site & Control & Control SE & Nitrogen & Nitrogen SE & Phosphorus & Phosphorus SE & $N+P$ & $N+P S E$ \\
\hline Duck & 66.8 & 9.6 & 54.9 & 7.3 & 74.2 & 11.9 & 80.3 & 18.4 \\
\hline S. Nest & 36.6 & 5.8 & 56.0 & 9.7 & 37.3 & 6.1 & 46.6 & 5.9 \\
\hline Bob Allen & 50.4 & 6.1 & 26.4 & 3.7 & 93.5 & 26.6 & 130.9 & 20.6 \\
\hline Rabbit & 268.7 & 32.8 & 239.0 & 14.1 & 198.7 & 17.9 & 217.2 & 27.0 \\
\hline Nine Mile & 265.5 & 32.2 & 305.6 & 17.4 & 188.8 & 21.7 & 230.4 & 14.5 \\
\hline Sprigger & 135.3 & 17.8 & 121.6 & 7.3 & 114.3 & 4.4 & 100.1 & 14.1 \\
\hline
\end{tabular}


Figure 3

\begin{tabular}{lcccccccc}
\multicolumn{2}{c}{ Soil organic carbon $(\mathrm{g} / \mathrm{m} 2)$} & & & & & & \\
Site & Control & Control SE & Nitrogen & Nitrogen SE & Phosphorus & Phosphorus SE & N+P & N+P SE \\
Duck & 2155.5 & 192.9 & 2175.5 & 124.3 & 1588.4 & 127.4 & 2200.5 & 191.3 \\
S. Nest & 1401.8 & 201.7 & 1303.2 & 143.4 & 1767.9 & 243.0 & 1329.3 & 248.5 \\
Bob Allen & 2533.6 & 141.1 & 2616.1 & 84.5 & 2808.0 & 120.9 & 3066.3 & 175.6 \\
Rabbit & 3260.0 & 399.0 & 3869.7 & 239.4 & 3421.1 & 312.6 & 2749.7 & 162.3 \\
Nine Mile & 2731.7 & 114.8 & 2972.4 & 219.6 & 2478.7 & 245.5 & 2804.7 & 47.9 \\
Sprigger & 2515.8 & 240.7 & 1794.0 & 288.6 & 1620.4 & 251.8 & 2177.9 & 203.1
\end{tabular}

Soil organic carbon (\%)

$\begin{array}{lcccccccc}\text { Site } & \text { Control } & \text { Control SE } & \text { Nitrogen } & \text { Nitrogen SE } & \text { Phosphorus } & \text { Phosphorus SE } & \text { N+P } & \text { N+P SE } \\ \text { Duck } & 1.4 & 0.1 & 1.6 & 0.1 & 1.0 & 0.1 & 1.3 & 0.1 \\ \text { S. Nest } & 0.9 & 0.1 & 1.0 & 0.1 & 1.2 & 0.1 & 0.9 & 0.1 \\ \text { Bob Allen } & 2.5 & 0.1 & 2.4 & 0.1 & 2.4 & 0.1 & 2.8 & 0.1 \\ \text { Rabbit } & 6.4 & 0.5 & 7.1 & 0.4 & 6.0 & 0.4 & 5.9 & 0.2 \\ \text { Nine Mile } & 4.9 & 0.2 & 5.2 & 0.2 & 4.5 & 0.2 & 4.9 & 0.2 \\ \text { Sprigger } & 2.1 & 0.1 & 1.6 & 0.1 & 1.5 & 0.1 & 1.8 & 0.2\end{array}$


Figure 4

\begin{tabular}{|c|c|c|c|}
\hline $\begin{array}{c}\text { aboveground } \\
\text { Thalassia leaf } \\
\text { N:P }\end{array}$ & $\begin{array}{c}\text { organic C } \\
\text { content } \\
(\mathrm{g} / \mathrm{m} 2)\end{array}$ & $\begin{array}{c}\text { Soil } \\
\text { organic C } \\
(\%)\end{array}$ & Site \\
\hline 141.9 & 2561.5 & 2.3 & $\mathrm{BA}$ \\
\hline \multirow[t]{2}{*}{98.8} & 2573.5 & 2.6 & $B A$ \\
\hline & 2649.9 & 2.3 & BA \\
\hline \multirow[t]{2}{*}{97.6} & 2036.2 & 2.6 & BA \\
\hline & 3067.9 & 2.5 & BA \\
\hline \multirow[t]{2}{*}{124.8} & 2312.5 & 2.6 & BA \\
\hline & 1454.5 & 1.1 & $D$ \\
\hline 80.8 & 2425.7 & 1.5 & $D$ \\
\hline 82.5 & 1696.8 & 1.3 & $D$ \\
\hline 82.9 & 2669.0 & 1.9 & $D$ \\
\hline 93.5 & 2400.8 & 1.3 & $D$ \\
\hline 98.2 & 2286.4 & 1.3 & $D$ \\
\hline 65.2 & 3101.4 & 4.8 & NM \\
\hline 53.3 & 3042.0 & 4.6 & NM \\
\hline 62.6 & 2713.3 & 5.9 & NM \\
\hline 65.5 & 2530.9 & 4.9 & NM \\
\hline 74.3 & 2412.8 & 4.6 & NM \\
\hline 72.2 & 2589.5 & 4.6 & NM \\
\hline 55.7 & 3951.3 & 6.2 & $\mathrm{R}$ \\
\hline 62.8 & 3625.5 & 5.1 & $\mathrm{R}$ \\
\hline 53.1 & 1612.0 & 6.3 & $\mathrm{R}$ \\
\hline 56.9 & 4321.5 & 7.1 & $\mathrm{R}$ \\
\hline 58.2 & 2699.4 & 5.6 & $\mathrm{R}$ \\
\hline 55.2 & 3350.6 & 8.3 & $\mathrm{R}$ \\
\hline 88.2 & 1354.8 & 0.9 & SN \\
\hline 94.3 & 650.0 & 0.6 & SN \\
\hline 91.4 & 2032.3 & 1.2 & SN \\
\hline 85.6 & 1066.0 & 0.8 & SN \\
\hline 128.7 & 1742.9 & 1.0 & SN \\
\hline 75.9 & 1565.0 & 0.9 & SN \\
\hline
\end{tabular}




\begin{tabular}{|c|c|c|c|}
\hline $\begin{array}{c}\text { Figure } 5 \\
\text { Total }\end{array}$ & & & \\
\hline Thalassia & organic C & Soil & \\
\hline$A b v C$ & content & organic C & \\
\hline$(\mathrm{g} / \mathrm{m} 2)$ & $(\mathrm{g} / \mathrm{m} 2)$ & (\%) & Site \\
\hline & 2561.5 & 2.3 & $\mathrm{BA}$ \\
\hline 6.1 & 2573.5 & 2.6 & $\mathrm{BA}$ \\
\hline 0.5 & 2649.9 & 2.3 & $\mathrm{BA}$ \\
\hline 3.1 & 2036.2 & 2.6 & $\mathrm{BA}$ \\
\hline 4.5 & 3067.9 & 2.5 & $\mathrm{BA}$ \\
\hline 9.9 & 2312.5 & 2.6 & $\mathrm{BA}$ \\
\hline & 1454.5 & 1.1 & $\mathrm{D}$ \\
\hline 6.9 & 2425.7 & 1.5 & $\mathrm{D}$ \\
\hline 2.2 & 1696.8 & 1.3 & $\mathrm{D}$ \\
\hline 6.8 & 2669.0 & 1.9 & $D$ \\
\hline 1.5 & 2400.8 & 1.3 & $D$ \\
\hline 4.8 & 2286.4 & 1.3 & $D$ \\
\hline 37.5 & 3101.4 & 4.8 & NM \\
\hline 16.1 & 3042.0 & 4.6 & NM \\
\hline 31.5 & 2713.3 & 5.9 & NM \\
\hline 32.8 & 2530.9 & 4.9 & NM \\
\hline 56.7 & 2412.8 & 4.6 & NM \\
\hline 15.9 & 2589.5 & 4.6 & NM \\
\hline 43.5 & 3951.3 & 6.2 & $\mathrm{R}$ \\
\hline 34.8 & 3625.5 & 5.1 & $\mathrm{R}$ \\
\hline 32.0 & 1612.0 & 6.3 & $\mathrm{R}$ \\
\hline 44.9 & 4321.5 & 7.1 & $\mathrm{R}$ \\
\hline 40.7 & 2699.4 & 5.6 & $\mathrm{R}$ \\
\hline 57.8 & 3350.6 & 8.3 & $\mathrm{R}$ \\
\hline 13.9 & 1354.8 & 0.9 & SN \\
\hline 17.0 & 650.0 & 0.6 & SN \\
\hline 9.2 & 2032.3 & 1.2 & SN \\
\hline 10.8 & 1066.0 & 0.8 & SN \\
\hline 5.2 & 1742.9 & 1.0 & SN \\
\hline 11.6 & 1565.0 & 0.9 & SN \\
\hline
\end{tabular}




\begin{tabular}{cccc}
$\begin{array}{c}\text { Figure } 6 \\
\text { Total }\end{array}$ & \multicolumn{3}{c}{} \\
Thalassia & organic C & \multicolumn{3}{c}{ Soil } & \\
Blw C & content & organic C \\
(g/m2) & (g/m2) & (\%) & Site \\
37.0 & 2561.5 & 2.3 & BA \\
59.2 & 2573.5 & 2.6 & BA \\
32.1 & 2649.9 & 2.3 & BA \\
53.2 & 2036.2 & 2.6 & BA \\
48.0 & 3067.9 & 2.5 & BA \\
72.7 & 2312.5 & 2.6 & BA \\
& 1454.5 & 1.1 & D \\
100.9 & 2425.7 & 1.5 & D \\
50.9 & 1696.8 & 1.3 & D \\
68.8 & 2669.0 & 1.9 & D \\
46.0 & 2400.8 & 1.3 & D \\
67.3 & 2286.4 & 1.3 & D \\
375.0 & 3101.4 & 4.8 & NM \\
172.0 & 3042.0 & 4.6 & NM \\
329.1 & 2713.3 & 5.9 & NM \\
192.9 & 2530.9 & 4.9 & NM \\
285.3 & 2412.8 & 4.6 & NM \\
238.9 & 2589.5 & 4.6 & NM \\
305.5 & 3951.3 & 6.2 & R \\
185.2 & 3625.5 & 5.1 & R \\
198.1 & 1612.0 & 6.3 & R \\
405.2 & 4321.5 & 7.1 & R \\
266.0 & 2699.4 & 5.6 & R \\
252.3 & 3350.6 & 8.3 & R \\
26.7 & 1354.8 & 0.9 & SN \\
60.7 & 650.0 & 0.6 & SN \\
47.2 & 2032.3 & 1.2 & SN \\
25.9 & 1066.0 & 0.8 & SN \\
30.0 & 1742.9 & 1.0 & SN \\
28.9 & 1565.0 & 0.9 & SN
\end{tabular}

